\title{
PressureDrop.jl: Pressure traverses and gas lift analysis for oil \& gas wells
}

\section{DOI: $10.21105 /$ joss. 01642}

\section{Software}

- Review ¿

- Repository ca

- Archive ¿

Submitted: 08 August 2019

Published: 08 November 2019

\section{License}

Authors of papers retain copyright and release the work under a Creative Commons Attribution 4.0 International License (CC-BY).

\section{Jared M. Noynaert ${ }^{1}$}

1 Alta Mesa Resources

\section{Summary}

For oil and gas wells, the pressure within the well (particularly the bottomhole pressure at the interface between the oil reservoir and the wellbore) is a key diagnostic measure, which can provide insight into current productivity, future potential, and properties of the reservoir rock from which the well is producing. Unfortunately, with the point of interest thousands of feet underground, regular direct measurement of bottomhole pressure is often prohibitively expensive or operationally burdensome.

The field of production engineering, which concerns itself with extracting hydrocarbons after the wells are drilled and completed, can in many cases be summarized as the practice of reducing flowing bottomhole pressure as economically as possible, since in most cases production is inversely proportional to bottomhole pressure. One of the methods used to accomplish this goal is gas lift, which changes operating states based on the pressure and temperature profile of the entire wellbore. As a result, designing and troubleshooting gas lift requires specific calculations based on the current wellbore conditions and the equipment utilized.

Due to the fundamental importance of pressure to all aspects of petroleum engineering, the calculation of multiphase pressure profiles using empirical correlations is a common task in research and practice, enabling diagnostics and transient analyses that otherwise depend on directly measured bottomhole pressure. Unfortunately, most options to perform these calculations depend on commerical software, and many software suites handle bulk calculations poorly or not at all. In addition, these commercial solutions typically have poor support for finding and modifying gas lift injection points for repeatedly modelling wells with that type of artificial lift (such as when examining variations in design, or conditions through time)

PressureDrop.jl is a Julia package for computing multiphase pressure profiles for gas lifted oil and gas wells, developed as an open-source alternative to feature subsets of nodal analysis or RTA software such as Prosper, Pipesim, or IHS Harmony. It currently calculates outlet-referenced models for producing wells using non-coupled temperature gradients using industry-standard pressure correlations: Beggs and Brill (Beggs \& Brill, 1973) with the Payne (Payne, Palmer, Brill, \& Beggs, 1979) correction, and Hagedorn and Brown (Brown, 1977) with the Griffith bubble flow (Griffith \& Wallis, 1961) correction, as well as the Ramey and Shiu temperature correlations (Ramey, 1962; Shiu \& Beggs, 1980). Output plots are generated using Gadfly.jl (Jones et al., 2019).

In addition to being open-source, PressureDrop.jl has several advantages over closedsource applications for its intended use cases: (1) it allows programmatic and scriptable use with native code, without having closed binaries reference limited configuration files; (2) it supports dynamic recalculation of injection points and temperature profiles through time; (3) it enables duplication and modification of models and scenarios, including dynamic generation of parameter ranges for sensitivity analysis and quantification of uncertainty; (4) PVT or pressure correlation options can be extended by adding functions in Julia code (or C, Python, or R); (5) it allows developing wellbore models from delimited input files or database records. 


\section{References}

Beggs, H. D., \& Brill, J. P. (1973). A Study of Two-Phase Flow in Inclined Pipes. Journal of Petroleum Technology, 25(5). doi:10.2118/4007-PA

Brown, K. E. (1977). The Technology of Artificial Lift Methods. Petroleum Publishing Co.

Griffith, P., \& Wallis, G. B. (1961). Two-Phase Slug Flow. J. of Heat Transfer, 83(3), 307-318. doi:10.1115/1.3682268

Jones, D. C., Arthur, B., Nagy, T., Mattriks, Gowda, S., Godisemo, Holy, T., et al. (2019, July). Giovineltalia/gadfly.jl: V1.1.0. doi:10.5281/zenodo.593105

Payne, G. A., Palmer, C. M., Brill, J. P., \& Beggs, H. D. (1979). Evaluation of Inclined-Pipe, Two-Phase Liquid Holdup and Pressure-Loss Correlations using Experimental Data. Journal of Petroleum Technology, 31(9). doi:10.2118/6874-PA

Ramey, H. J. (1962). Wellbore Heat Transmission. Journal of Petroleum Technology, 14(4). doi:10.2118/96-PA

Shiu, K. C., \& Beggs, H. D. (1980). Predicting temperatures in flowing oil wells. Journal of Energy Resources Technology, 102(1). doi:10.1115/1.3227845 